\title{
Pitching Moment Caused by Rotor Flow Interference on a Small Quad-rotor Unmanned Aerial Vehicle in a Uniform Flow*
}

\author{
Hikaru OTSUKA $^{1) \dagger}$ and Keiji NAGATAN ${ }^{2)}$ \\ ${ }^{1)}$ Department of Aerospace Engineering, Tohoku University, Sendai, Miyagi 980-8579, Japan \\ ${ }^{2}$ New Industry Creation Hatchery Center, Tohoku University, Sendai, Miyagi 980-8579, Japan
}

\begin{abstract}
Multi-rotor unmanned aerial vehicles (UAVs) are used in various applications because they can hover and take off and land vertically. Although they are useful for civilian applications, their cruise flight speeds are limited owing to the generation of a pitching moment in a uniform flow. However, aerodynamic forces acting on multi-rotor UAVs have not been precisely examined. The effects of the body-yaw angle, body-pitch angle, and distance between rotors on the pitching moment and thrust in a uniform flow are important considerations when designing UAVs. Furthermore, rotor flow interaction can affect the rotor performance in horizontal flight. The objective of the present study is to examine the thrust and pitching moment of a quad-rotor UAV in a uniform flow using different configurations. First, the rotor thrust and pitching moment on two rotors were measured while changing the relative rotor positions in a wind tunnel. Second, the thrust and moment of a quad-rotor UAV were measured at different yaw directions, pitch angles, and rotors distances. Experiment results clarify that maintaining the yaw direction in a plus configuration, rather than an $\mathrm{X}$ configuration, with regard to the flow direction can reduce the pitching moment.
\end{abstract}

Key Words: Flow Interaction, Multi-rotor UAV, Pitching Moment, Wind Tunnel Experiment, VTOL

\section{Nomenclature}

$A$ : rotor disk area, $\pi R^{2}, \mathrm{~m}^{2}$

$R$ : rotor radius, $\mathrm{m}$

$C t$ : thrust coefficient, $T / \rho A(\Omega R)^{2}$

$C_{M y}$ : moment coefficient, $M_{y} / \rho A R(\Omega R)^{2}$

$M_{y}$ : pitching moment, $\mathrm{N} \cdot \mathrm{m}$

$T$ : thrust, $\mathrm{N}$

$\rho$ : atmospheric density, $\mathrm{kg} / \mathrm{m}^{3}$

$\Omega$ : rotor angular velocity, $\mathrm{rad} / \mathrm{s}$

\section{Introduction}

Small multi-rotor unmanned aerial vehicles (UAVs) are aircraft with multiple rotors, and are capable of vertical take-off and landing (VTOL). ${ }^{1)}$ Owing to the development of small electronic speed controllers and high-capacity batteries, small multiple rotors are implemented and used in small VTOL UAVs, which can hover and begin flight without requiring long runway. Therefore, UAVs have been used for various tasks, including the inspection of old bridges, walls and wind turbines, as well as topographical surveys and the transport of medical kits.

Although small multi-rotor UAVs have VTOL functionality, wind speeds under which they can fly are limited compared to fixed-wing aircrafts. Therefore, UAV operations

(C) 2018 The Japan Society for Aeronautical and Space Sciences

*Presented at the 55th Aircraft Symposium, 20-22 November 2017, Matsue, Japan

Received 29 August 2017; final revision received 28 November 2017; accepted for publication 25 December 2017.

†Corresponding author, h-otsuka@frl.mech.tohoku.ac.jp are frequently canceled because of strong winds. To improve multi-rotor UAV usability outdoors, the ability to fly under fast wind conditions is a necessity.

During horizontal flight, multi-rotor UAVs tilt forward to generate thrust in the direction of motion. Their rotors generate thrust to support their body weight, therefore enabling horizontal movement. Thus, the rotor speeds are accelerated during horizonal flight compared to when hovering under windless conditions. In addition, a head-up pitching moment is generated on the body during flight. The UAVs cancel such moments, creating a difference in thrust between the front and rear sides in the flight direction. The rear rotors are accelerated, and approach the rotor speed limitation. This means that flight speed limitation is reduced as a result of the pitching moment. Hence, reducing the generation of pitching moment has the potential to improve the wind speed limitation during outdoor flight.

One factor with regard to pitching moment generation seems to be rotor wake interference between the rotors on multi-rotor UAVs. Numerical and experimental studies on the effects of rotor flow interference have been conducted using large-scale helicopters that can be manned by a pilot. $^{2-8)}$ However, few studies have focused on the effects of rotor flow interface on the aerodynamic force acting on small multi-rotor UAVs in a flow. For the large-scale helicopters, the effects of the rotor flow interference were determined for the development of tandem-rotor helicopters. ${ }^{2,3)}$ These helicopters were designed to prevent the occurrence of rotor flow interference with a difference in rotor height between the front and rear rotors. The effects of the flow interference in the two rotors have also been summarized. ${ }^{4,5}$ Moreover, for the large-scale tandem-rotor and multi-rotor 
helicopters, numerical studies were conducted focusing on the rotor wake and its interference. ${ }^{6-8)}$ For the rotor interference of small multi-rotor UAVs, Ramasamy examined the hovering performance of a quad-rotor UAV in relation to various configurations under windless conditions. ${ }^{9)}$ Theys et al. determined the performance of a small isolated rotor in an oblique flow using a wind tunnel to estimate the performance of small multi-rotor UAVs during flight. ${ }^{10)}$ However, the rotors of an in-flight multi-rotor UAV cause rotor flow interference. The effect of the rotor flow interference differs from the hovering that occurs under windless conditions. Therefore, studies on rotor flow interference in a uniform flow are required to examine the performance of an in-flight multi-rotor UAV. Russell et al. conducted aerodynamic force measurements on several commercial multirotor UAVs using a low-speed wind tunnel. ${ }^{11)}$ Although their study elucidated the aerodynamic force on the UAVs, the difference in force was not examined in relation to the configuration based on the rotor distance, or body yaw with respect to the flow direction. Therefore, evaluation of the pitching moment generated by a multi-rotor UAV in relation to the rotor configuration is required to reduce this moment in order to design multi-rotor UAVs that can fly under high-speed wind conditions.

For the pitching moment in different yaw directions, Hwang et al. conducted a numerical investigation on a quad-rotor in a uniform flow. ${ }^{12)}$ They noted that rotor flow interaction increases the pitching moment of the body, and that a rotor configuration resembling an X-shape in the flow direction generates a higher pitching moment than other body yaw directions. Their calculation results suggest that a rotor configuration resembling a plus-shape reduces the pitching moment. We assumed that changing the rotor distance and yaw direction would reduce the pitching moment by avoiding a flow interaction. Therefore, it is important to experimentally verify effects of body configuration on the generation of the pitching moment.

As mentioned above, the pitching moment on small multirotor UAVs in flight reduces flight speed limitations. Therefore, reducing the pitching moment is assumed to improve the endurance of UAVs. Hwang et al. conducted numerical analyses and reported that rotor flow interference increases the moment and a rotor configuration that avoids interference reduces the moment. ${ }^{12)}$ However, the pitching moment of UAVs with different rotor configurations in flows was not clarified experimentally. The objective of this study is to examine the difference in pitching moment generated on a quad-rotor UAV with different rotor configurations while flying in a uniform flow. Such an evaluation will enable us to design a quad-rotor UAV that generates lower pitching moment during horizontal flight. We focused on a quad-rotor UAV because it is a basic type of small multi-rotor vehicle. In this study, first, the rotor thrust and pitching moment in a flow interaction between two rotors were evaluated by changing their relative positions in a wind tunnel. The experiments were aimed at examining the effects of rotor flow interference on rotor performance in relation to the basic relative positions. Second, pitching moment generated on a small quad-rotor UAV was evaluated by changing the rotor distance, pitch angle, or yaw direction, in a free-stream.

\section{Flow Interaction between Two Rotors}

The rotor flow interaction between two rotors was examined using a low-speed wind tunnel. The experiment aimed at examining the effects of rotor flow interaction under a basic rotor situation of a quad-rotor UAV flying in a uniform flow. The relative positions of the rotors were changed in height, depth normal to the flow direction, and distance along the flow direction. In the measurement, the pitch angle of the rotor plane was changed because the rotors tilt during horizontal flight, and the interaction is considered to depend on the pitch angle.

\subsection{Methodology}

The wind tunnel was a semi-closed circuit. Figure 1 shows the experimental setup in the wind tunnel. The rotor radius used in the experiment was $119 \mathrm{~mm}$, as shown in Fig. 2. The rotor solidity was 0.105 . The rotor utilized is one that is used for commercial multi-rotor UAVs. The rotor speed was controlled using an electronic speed controller. The rotor speed was set to 6,000 rpm during the experiments. The Reynolds number of the cord length at a $75 \%$ blade span was $5.4 \times 10^{5}$. During the measurements, air in the wind tunnel leaked from the test section owing to the rotor wake. Thus, the speed of the free-stream was degraded. To keep a constant flow speed, the free-stream speed was maintained at $8 \mathrm{~m} / \mathrm{s}$ while adjusting the speed of the wind tunnel fan. The test section shape was an octagon, with a 1,010-mm length between opposite sides.



Fig. 1. Experimental setup in the wind tunnel.

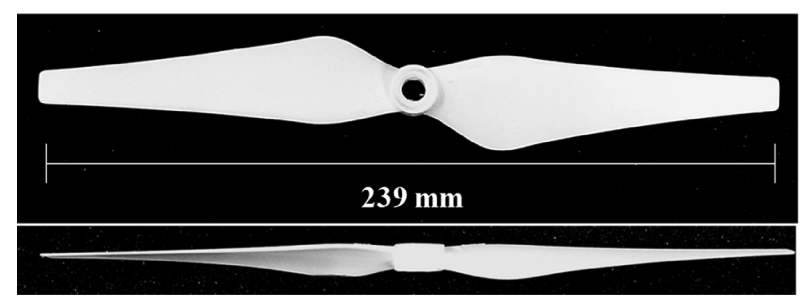

Fig. 2. Experimental rotor. 
Figure 3 shows the definition of rotor placement for all measurement conditions. Figure 4 shows the detailed placement for each condition. Table 1 lists the experimental conditions. The support pillar of the front rotor was moved using a single-axis liner guide actuated using a motor. The position of the rotor was adjusted with a resolution of $1 \mathrm{~mm}$. The range of geometric parameters was changed focusing on the rotor placement on a small quad-rotor UAV. The length was expressed as a dimensionless value based on the rotor radius.

The average force based on 5,000 samples was recorded for each measurement. Three measurements were taken and the average of those measurements was used in the results. The specifications of a 6-axis force sensor are listed in Table 2. Figure 4 shows the position of the sensor and the definition of the rotor pitch angle in Fig. 5.

The atmospheric density during the five-day experiment was $1.16 \mathrm{~kg} / \mathrm{m}^{3}$.

\subsection{Results}

\subsubsection{Rotor distance and pitch angle}

The thrust and pitching moment were measured by changing the rotor distance between the front and rear rotors, and

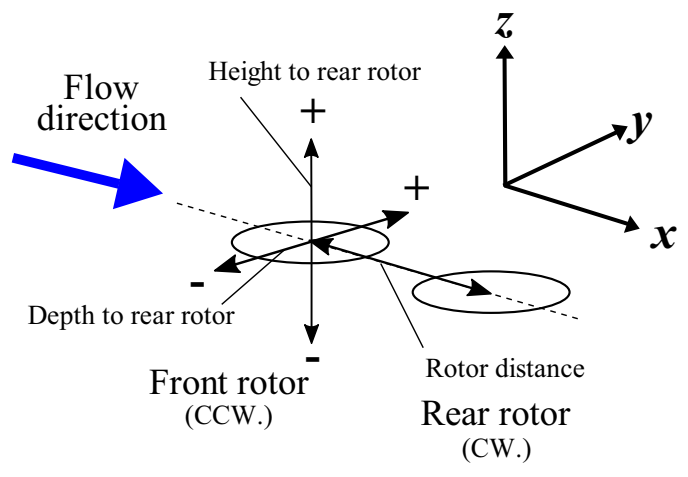

Fig. 3. Positions of rotors for each geometry. the rotor plane pitch angles. During horizontal flight of a quad-rotor UAV, the body tilts in the moving direction to generate thrust from the rotors. Thus, an evaluation of flow intrafraction was conducted by changing the rotor pitch angle. The thrust coefficient and pitching moment coefficient of the front and rear rotors are shown in Figs. 6-9. The thrust and pitching moment of the isolated rotor are shown with the broken lines in the figures. A sensor offset from the center of the rotor generates an additional pitching moment because of rotor drag. To examine the pure pitching moment at the center of the rotor, the moment caused by the drag force was subtracted from the pitching moment measured using Eq. (1).

$$
M_{y}=\text { Measured } M_{y}-\text { drag force } \times \text { sensor offset }
$$

For the front rotor, increasing the rotor distance has no effect on front rotor performance, as shown in Figs. 6 and 7. Moreover, the thrust and pitching moment at all pitch angles are close to those of an isolated rotor.

For the rear rotor, the thrust and pitching moment decrease as the rotor distance is reduced, as shown in Figs. 8 and 9. At a rotor plane pitch angle of $90^{\circ}$ and rotor distance of $2.1 R$, the thrust and pitching moment are $8.3 \%$ and $57 \%$ lower, respectively, compared to the moments of an isolated rotor.

\subsubsection{Height}

The thrust coefficient and pitching moment coefficient of a rear rotor with a change in relative height of the front rotor to the rear rotor being a rotor distance $3.0 R$ are shown in Figs. 10 and 11, respectively. As shown in Fig. 11, if the height of the front rotor is lower than the rear rotor by $-2.0 R$, or higher than $2.0 R$, the rear rotor thrust is close to $3.4 \mathrm{~N}$, which is almost the same as that of the isolated rotor thrust. The thrust at a height of $1.0 R$ to $1.5 R$ was the smallest within the height range for each rotor distance. The rear rotor thrust at a height of $1.0 R$ decreased by $30 \%$ from $3.4 \mathrm{~N}$. As

Table 1. Conditions of two-rotor measurement.

\begin{tabular}{|c|c|c|c|c|c|c|c|}
\hline \multirow{2}{*}{ Target geometry } & \multirow{2}{*}{$\begin{array}{c}\text { Flow speed } \\
\mathrm{m} / \mathrm{s}\end{array}$} & \multirow{2}{*}{$\begin{array}{c}\text { Rotor speed } \\
\text { rpm }\end{array}$} & Distance & Depth & Height & \multirow{2}{*}{ Pitch angle } & \multirow{2}{*}{ Measured rotor } \\
\hline & & & \multicolumn{3}{|c|}{$R$ (Radius: $119 \mathrm{~mm}$ ) } & & \\
\hline 1. Distance & 8 & 6,000 & $2.1,3,4,5,6$ & 0 & 0 & $60-100$ & Front*2, rear \\
\hline 2. Depth & 8 & 6,000 & 3 & $-3-3^{* 1}$ & $0,1,2$ & 90 & Rear \\
\hline 3. Height & 8 & 6,000 & 3 & 0 & $-2-3$ & 90 & Rear \\
\hline 4. Isolated rotor & 8 & 6,000 & $\mathrm{~N} / \mathrm{A}$ & $\mathrm{N} / \mathrm{A}$ & $\mathrm{N} / \mathrm{A}$ & $60-100$ & $\mathrm{~N} / \mathrm{A}$ \\
\hline
\end{tabular}

${ }^{* 1}$ Depth range is from $-1.5 R$ to $1.5 R$ for a height of $2.0 R$, and a distance of $5.0 R$.

${ }^{* 2}$ Front rotor direction is counter-clockwise. Rear rotor direction is clockwise from top view.

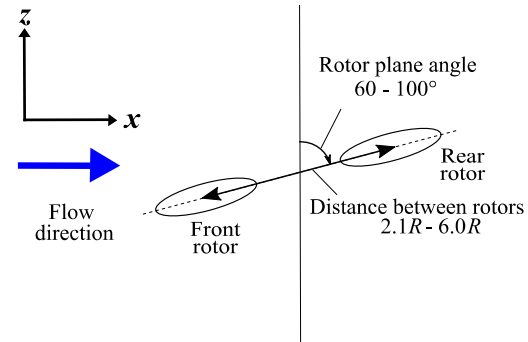

(a) Distance and pitch angle

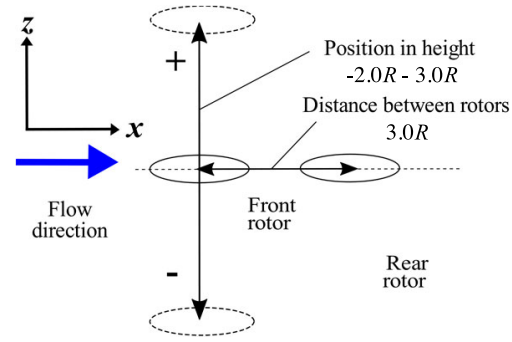

(b) Height

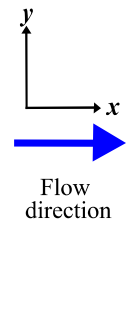

Fig. 4. Detailed position of rotors under each experimental condition. 
Table 2. Specifications of force sensor.

\begin{tabular}{cccccc}
\hline & $\begin{array}{c}\text { Product } \\
\text { number }\end{array}$ & $\begin{array}{c}\text { Force } \\
\text { range } \\
\mathrm{N}\end{array}$ & $\begin{array}{c}\text { Moment } \\
\text { range } \\
\mathrm{N} \cdot \mathrm{m}\end{array}$ & Resolution & $\begin{array}{c}\text { Sampling } \\
\text { rate } \\
\mathrm{Hz}\end{array}$ \\
\hline $\begin{array}{c}\text { Isolated rotor } \\
\text { measurement }\end{array}$ & $\begin{array}{c}\text { SFS0017 } \\
\text { F100M0 } \\
\text { R5U6 }\end{array}$ & \pm 10 & \pm 0.5 & $1 / 1,000$ & 1,200 \\
& & & & \\
\hline $\begin{array}{c}\text { Quad-rotor } \\
\text { measurement }\end{array}$ & CFS034 & \pm 100 & \pm 2.0 & $1 / 2,000$ & 1,200 \\
\hline
\end{tabular}
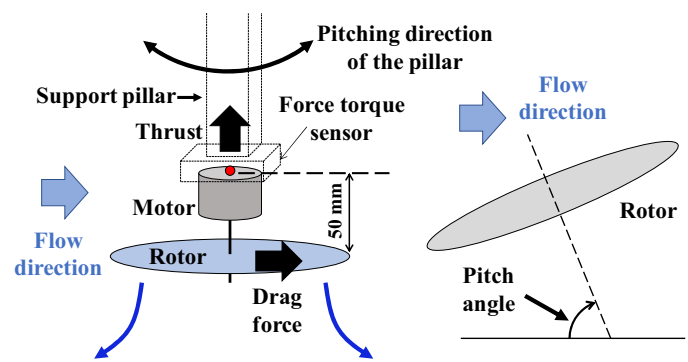

Fig. 5. Sensor position and definition of rotor pitch angle.

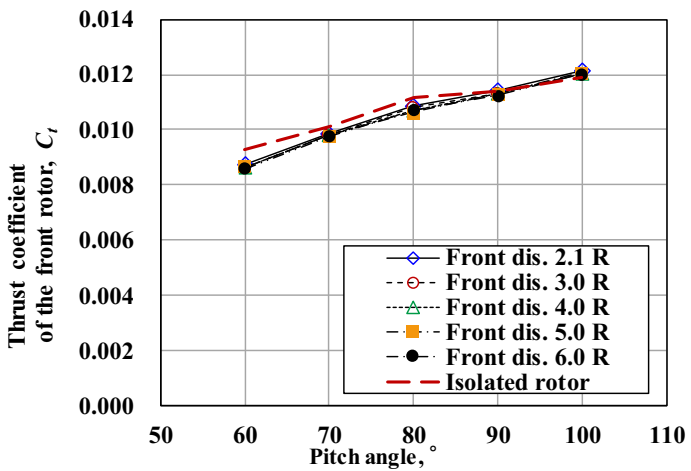

Fig. 6. Thrust of front rotor with change in rotor distance.

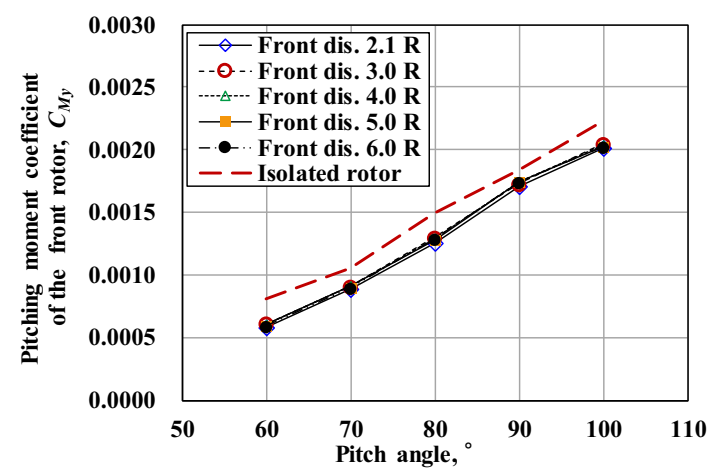

Fig. 7. Pitching moment of front rotor with change in rotor distance.

shown in Fig. 12, the pitching moment has two minimum points at $0.5 R$ and $2.0 R$ within range of the heights during the experiment. The rear rotor pitching moment decreases by $55 \%$ at a height of $0.5 R$, and by $30 \%$ at a height of $2.0 R$, from $6.4 \times 10^{-2} \mathrm{~N} \cdot \mathrm{m}$, which was the same as the pitching moment of the isolated rotor.

\subsubsection{Depth}

The thrust coefficient and pitching moment coefficient of the rear rotor with a change in the depth position of the front

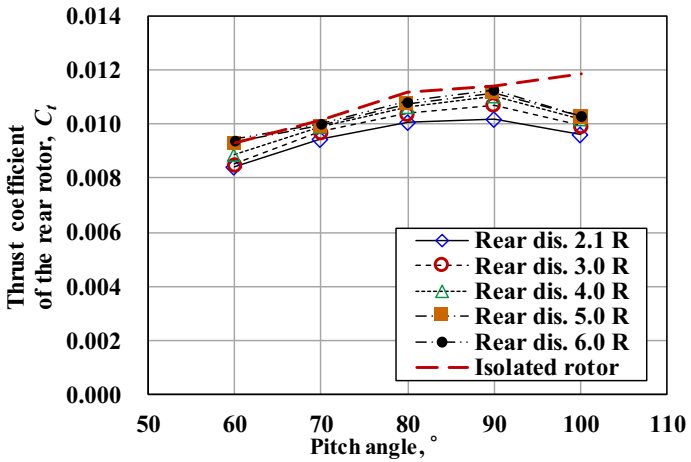

Fig. 8. Thrust of rear rotor with change in rotor distance.

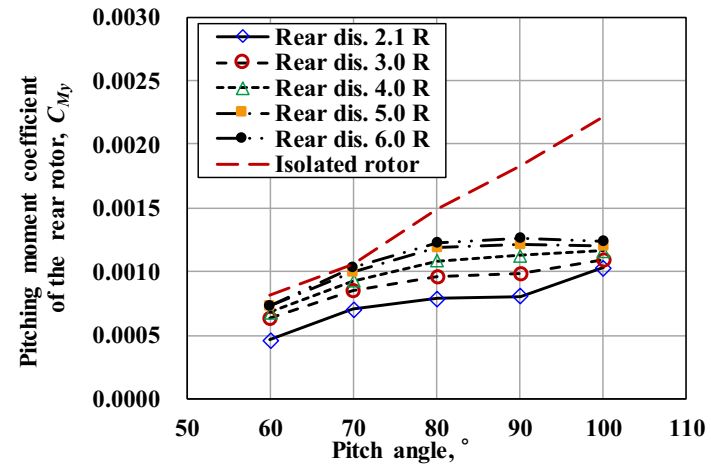

Fig. 9. Pitching moment of rear rotor with change in rotor distance.

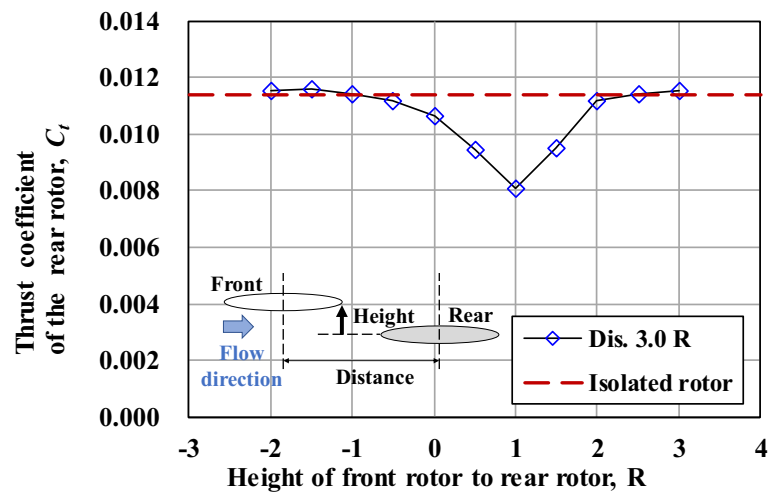

Fig. 10. Thrust of rear rotor with change in height.

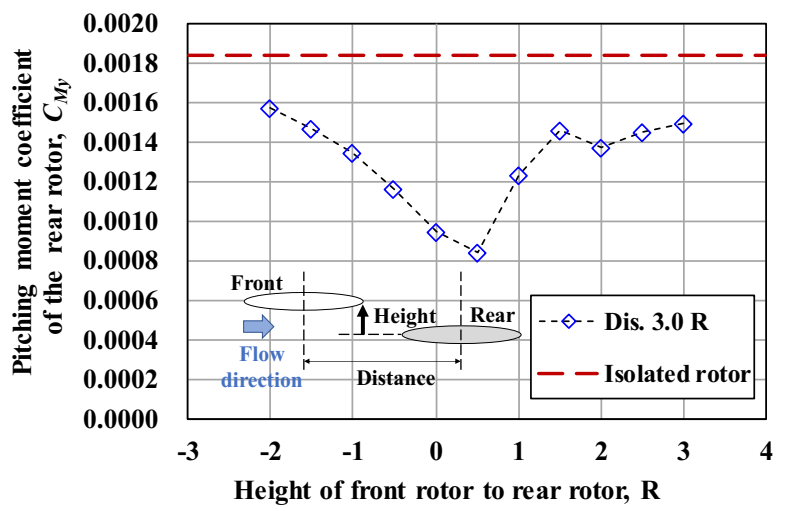

Fig. 11. Pitching moment of rear rotor with change in height. 


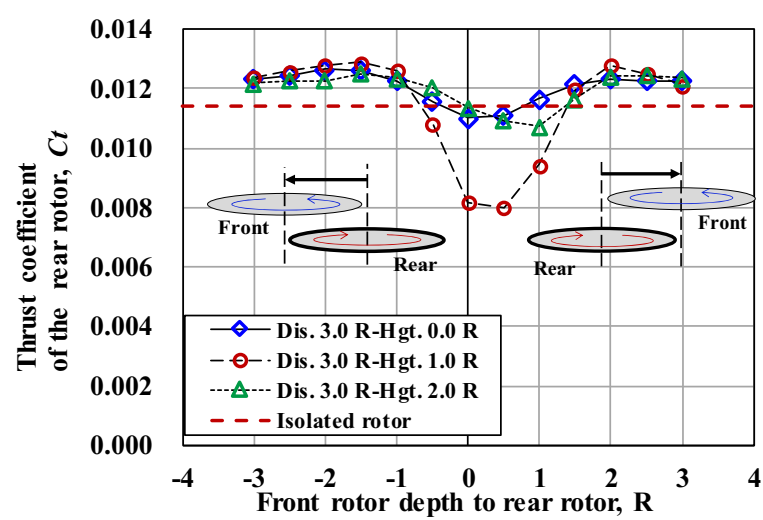

Fig. 12. Thrust of the rear rotor with change in depth.

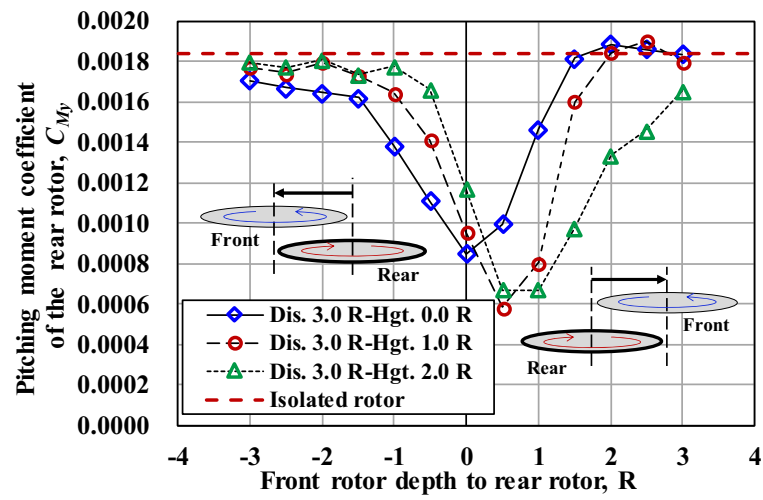

Fig. 13. Pitching moment of the rear rotor with change in depth.

rotor are shown in Figs. 12 and 13, respectively. In the measurements, the rotor distance was $3.0 R$. The height was changed to $0.0 R, 1.0 R$, and $2.0 R$.

Thrust was the smallest near a depth of $0.5 R$ for the three heights shown in Fig. 12. At a depth of $0.5 R$, the front rotor is on the right side of the rear rotor. During the experiment, the rear rotor rotation direction was clockwise. For the three heights, the thrust was close to $3.6 \mathrm{~N}$, which was higher than that of the isolated rotor thrust, when the front rotor depth was either under or over $2.0 R$. At a depth of $0.5 R$ and a height of $1.0 R$, the thrust decreased by $1.3 \mathrm{~N}$, from $3.6 \mathrm{~N}$. However, at a depth of $0.5 R$ and a height of $0.0 R$ or $2.0 R$, the loss of thrust was less than $0.3 \mathrm{~N}$. In the experiment, the thrust at depth of $0.5 R$ was the smallest at a height of $1.0 R$ in the three heights. The loss of thrust as depth changes is significantly influenced by the height.

For the pitching moment shown in Fig. 13, the depth position of the minimum pitching moment moved from $0.0 R$ to $1.0 R$ as the height increased from $0.0 R$ to $3.0 R$. An increase in depth means rightward movement of the rear rotor in the depth direction. In contrast to thrust, the minimum value of the pitching moment at each height decreased as the height increased. At a height of $0.0 R$, the depth of the minimum moment was $0.0 R$, and the position of the rotors coincided in the depth direction.

\subsection{Discussions}

\subsubsection{Distance and pitch angle}

In previous studies, it was elucidated that the velocity in- duced as the flow passes the rotor disk decreases the thrust. ${ }^{13)}$ The rear rotor thrust and pitching moment are presumed to be affected by the front rotor wake between the two rotors. In contrast, the front rotor is presumed to be outside of the effective zone of rotor interference. The experiment results show that the front rotor thrust is independent of rotor distance. Therefore, the flow interaction between the two rotors only interferes with the rear rotor.

The thrust and pithing moment decrease as rotor distance increases. Although the pitching moment of the rear rotor changes based on the rotor flow interference, the degradation in the rotor pitching moment is lower than $0.03 \mathrm{~N} \cdot \mathrm{m}$. Therefore, we regard the difference in thrust between rotors as having a more significant effect on the head-up pitching moment of a quad-rotor UAV than the summation of degradation in the rotor pitching moment.

In addition, tilting the rotor plane to a lower angle attenuates the effects of the rotor flow interference on the thrust and pitching moment. When considering the horizontal flight of a quad-rotor UAV, the pitch angle of the UAV inclines toward the forward direction as the flight speed is accelerated. Thus, the effect of the rotor flow interference seems to be reduced as flight speed increases.

In short, we verified that rear thrust decreases because of the rotor flow interference that occurs as the distance between the two rotors is reduced. Moreover, the degradation in thrust was negligible for a rotor distance of greater than $5.0 R$. Thus, a reduction in pitching moment can be expected as rotor distance increases.

\subsubsection{Height}

We deduced that the thrust is smallest at a height of $1.0 R$ or $1.5 R$ at most because the front rotor wake flows directly into the rear rotor directly.

When the front rotor is lower than the rear rotor, the rear rotor thrust increases slightly as the front rotor moves to a lower height. The effect of the rotor flow interference when the front rotor is lower than the rear rotor appears to be smaller than when the front rotor is higher than the rear rotor.

The experiment results prove that lowering the front rotor height prevents rotor interference in a uniform flow. The placement of rotors with a lower front rotor is the same that for a tandem-rotor helicopter design. In terms of the flight of a quad-rotor UAV, our findings suggest that the effect of such interference is small because inclining the body toward an upwind direction makes the front rotor position lower than that of the rear rotor.

\subsubsection{Depth}

In terms of changing the depth, the loss of thrust of the rear rotor caused by flow interaction is significant when the depth is $-1.0 R$ to $2.0 R$. As for the effect on the pitching moment, maintaining the interval over the rotor diameter avoids changing pitching moment at the same rotor height. Thus, when designing the rotor placement of a quad-rotor, maintaining the interval of the rotors in the depth direction over the rotor diameter can prevent the effects of a rotor flow interaction.

However, as the height of the front rotor increases, the depth range under the rotor interference moves toward the 


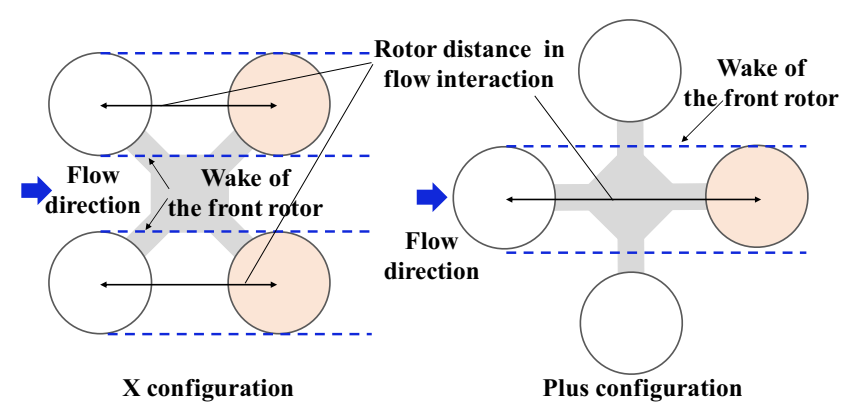

Fig. 14. Wake of the front rotors under plus- and X-shaped configurations.

right side of the rear rotor, which rotates in a clockwise direction. When the front rotors are higher than the rear rotor, a shift in the affected depth range toward the right side of the rear rotor with clockwise rotation is required when considering rotor placement design.

The largest decrease in pitching moment that occurred when changing the depth while maintaining the same rotor height was found to be $0.03 \mathrm{~N} \cdot \mathrm{m}$. In addition, the largest degradation of thrust was $1.0 \mathrm{~N}$. In terms of generating pitching moment on a quad-rotor in horizontal flight, the effect of the thrust difference on the moment is stronger than the effect of pitching moment degradation.

\subsubsection{Effect of rotor flow interference on quad-rotors}

In the two-rotor experiments, we verified the effect of rotor flow interaction on the rotor thrust and pitching moment for three positional relationships. The results indicate that preventing flow interference reduces the loss of thrust at the rear rotors. Therefore, we deduced that preventing such interference reduces the pitching moment of the quad-rotor.

In terms of rotor placement of the quad-rotors, the rotors are at the same plane and the difference in height is determined by only the posture of the quad-rotor body. Therefore, the depth and rotor distance affect the reduction in pitching moment of the quad-rotor during horizontal flight. When the rotor distance approaches but does not exceed 3.0R, the effect of the flow interference is presumed to be significant. In the design process, expanding the rotor distance is suitable for reducing the pitching moment. Moreover, the distance between rotors along the flow direction depends on the yaw direction of the quad-rotors. When the body configuration toward the flow direction is in a plus shape, rather than an $\mathrm{X}$ shape, as shown in Fig. 14, a reduction in pitching moment can be expected. Because the number of rear rotors in the front rotor wake is reduced and the distance between the rotors along the flow direction is longer than that in the yaw direction, preventing the occurrence of rotor flow interference results in a reduction in pitching moment.

\section{Quad-rotor Measurement}

To examine the thrust, pitching moment, and force along the $x$-axis $(F x)$ of the body coordinate of a quad-rotor $\mathrm{UAV}$ in horizontal flight, the forces were measured in the wind tunnel. During the experiment, we examined the differences in forces when changing the body pitch angles, yaw di-

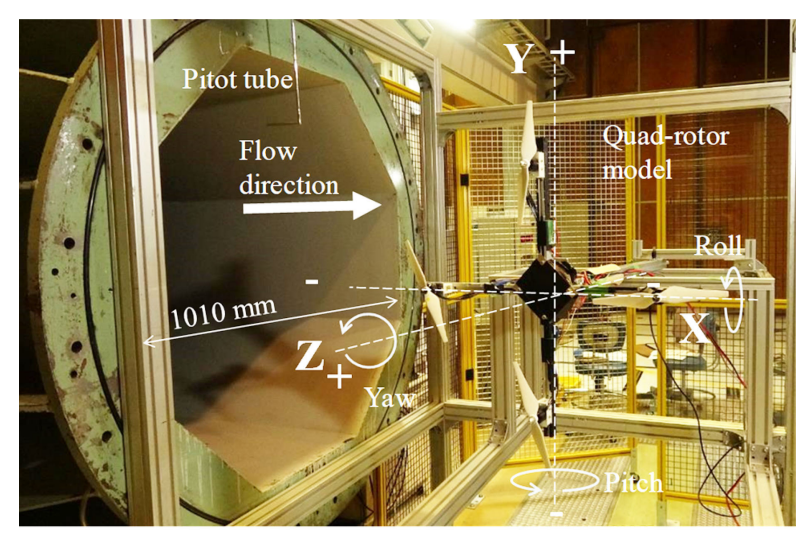

Fig. 15. Experiment setup of a quad-rotor model in a wind tunnel.

Table 3. Conditions of quad-rotor measurement.

\begin{tabular}{lccccc}
\hline & $\begin{array}{c}\text { Flow } \\
\text { speed } \\
\mathrm{m} / \mathrm{s}\end{array}$ & $\begin{array}{c}\text { Rotor } \\
\text { speed } \\
\mathrm{rpm}\end{array}$ & $\begin{array}{c}\text { Distance } \\
\text { between } \\
\text { rotors } \\
R(119 \mathrm{~mm})\end{array}$ & $\begin{array}{c}\text { Pitch angle } \\
\circ\end{array}$ & $\begin{array}{c}\text { Yaw angle } \\
\circ\end{array}$ \\
\hline Quad-rotor & 8 & 6,000 & $2.1,3,4,5,6$ & $60,70,80,90$ & $0,45,90$ \\
\hline
\end{tabular}

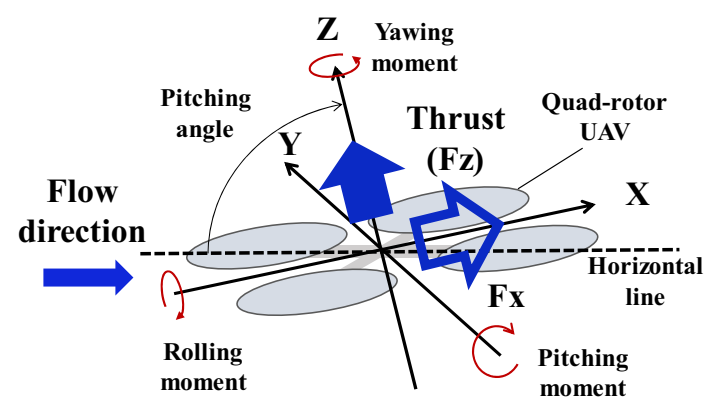

Fig. 16. Definition of force coordinates on the quad-rotor model.

rection, and rotor distance. We aimed to verify measurement of pitching moment reduction by changing the rotor distance and yaw direction.

\subsection{Methodology}

We measured the thrust, pitching moment, and $F x$ when changing the rotor distance and yaw in the direction of motion. The experimental setup of the quad-rotor model is shown in Fig. 15. The definition of force on a quad-rotor model is shown in Fig. 16. Experiment conditions are shown in Table 3. Moreover, the top views of the model for each configuration and yaw direction are shown in Figs. 17 and 18. The pitch angle of the model changed from 60 to $90^{\circ}$ with an increment of $10^{\circ}$. The rotor distance was changed from $2.1 R$ to $3.0 R$. The distance of $2.1 R$ was close to the diameter of the rotor. Therefore, the distances between the rotor blade tips were small. The yaw direction of the model was changed to 0,45 , and $90^{\circ}$. The rotors of a quad-rotor rotate in the opposite direction of the adjoining rotors. Therefore, the rotational direction of the rotor is different between the yaw angles 0 and $90^{\circ}$. The specifications of the force torque sensor are shown in Table 2. The forces were measured three times under the same conditions in a similar way to the measurements of the two rotors. 

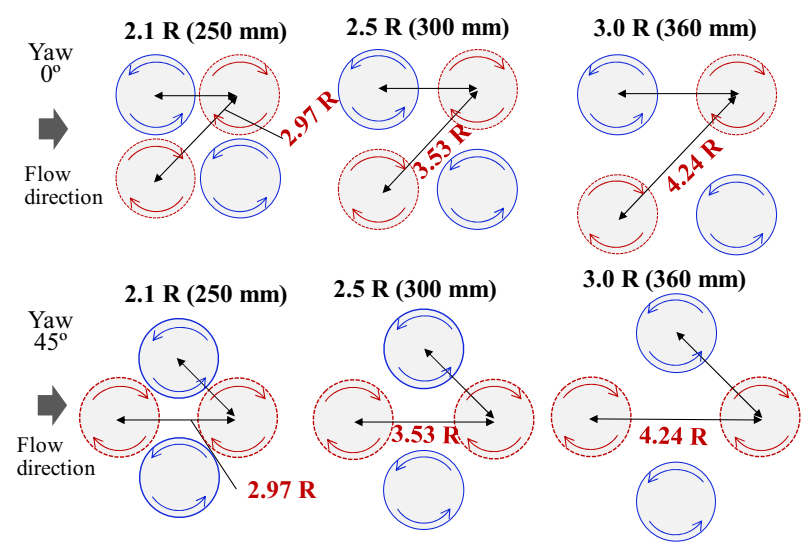

Fig. 17. Top view of quad-rotor for each configuration.
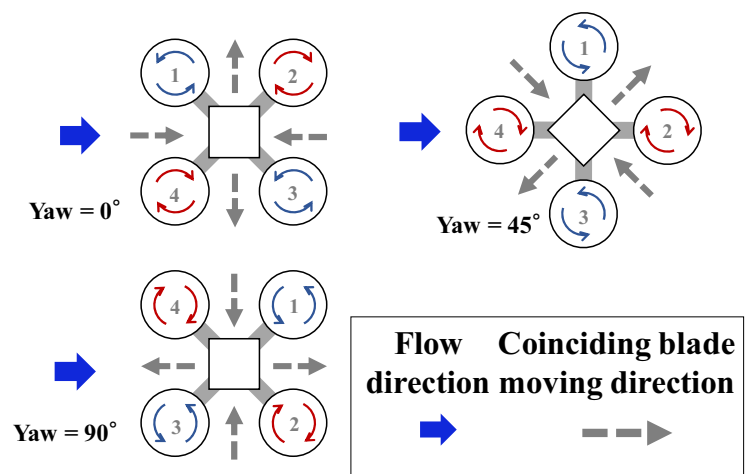

Fig. 18. Definition of yaw direction.

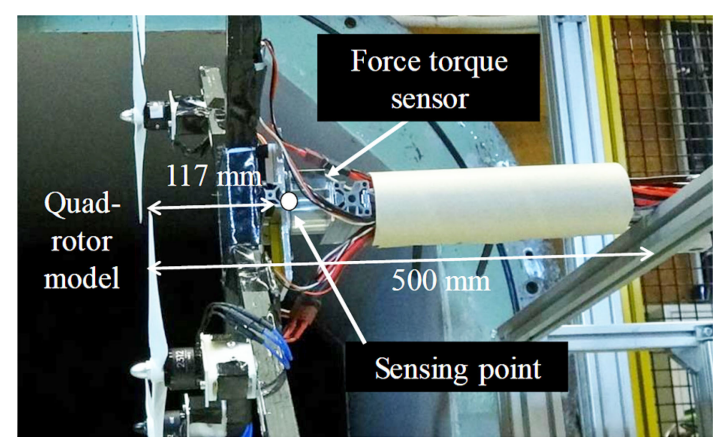

Fig. 19. Attached force torque sensor under a quad-rotor model.

The position where the sensor was attached is shown in Fig. 19. The sensor was attached to the bottom of the model, not at the center of gravity. Thus, the pitching moment measured contains the drag force on the body, which increases the pitching moment. We can compare the difference in pitching moment using the forces measured in the test results. However, a comparison with a different study requires consideration of an offset sensor position.

\subsection{Results}

\subsubsection{Comparison of different yaw directions}

The thrust, $F x$, and pitching moment in different yaw directions are shown in Figs. 20-24. The thrust is expressed using the thrust coefficient. The pitching moment is expressed using the moment coefficient. For a quad-rotor model, the thrust and moment coefficient are expressed using

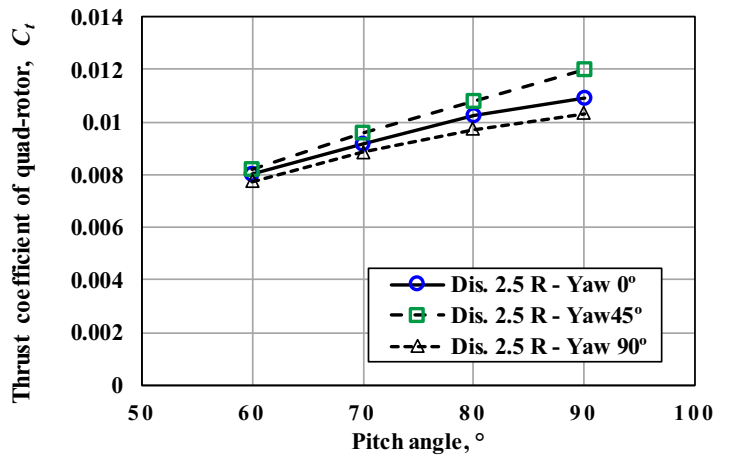

Fig. 20. Thrust of a quad-rotor at different yaw angles.

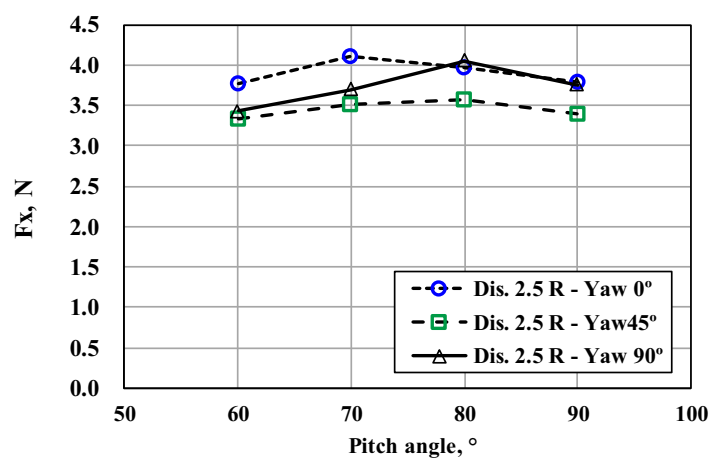

Fig. 21. Fx of a quad-rotor at different yaw angles.

Eqs. (2) and (3) in this study.

$$
C_{T}=\text { Thrust of all rotors } / 4 \rho A R(\Omega R)^{2}
$$

$C_{M y}=$ Pitching moment of the model $/ \rho A R(\Omega R)^{2}$

As shown in Fig. 20, the thrust increased as pitch angle increased in the three yaw directions. Thrust at a yaw angle of $45^{\circ}$ was the largest among the yaw directions. The thrust at a yaw angle of $45^{\circ}$ and pitch angle of $90^{\circ}$ was $14.1 \mathrm{~N}$, at a yaw angle of $0^{\circ}$ was $12.9 \mathrm{~N}$, and at a yaw angle of $90^{\circ}$ was $12.1 \mathrm{~N}$. The difference in thrust at the pitch angle was $2 \mathrm{~N}$. The difference in thrust between the yaw directions decreased as pitch angle decreased. At a yaw angle of $45^{\circ}$ and pitch angle of $60^{\circ}$, the thrust was $9.6 \mathrm{~N}$. At a yaw angle of $90^{\circ}$, the thrust was $9.1 \mathrm{~N}$. The difference in thrust between the yaw directions was $0.5 \mathrm{~N}$. This difference was smaller than that at pitch angle of $90^{\circ}$.

The $F x$ was from 3.5 to $4.1 \mathrm{~N}$, as shown in Fig. 21. The difference in change in the pitch angle from the $F x$ was smaller than $0.5 \mathrm{~N}$.

For the pitching moment shown in Fig. 22, the moment was the smallest at a yaw angle of $45^{\circ}$, i.e., $0.48 \mathrm{~N} \cdot \mathrm{m}$. Pitching moments at 0 and $90^{\circ}$ were almost the same. The pitching moment at a yaw angle of $0^{\circ}$ and pitch angle of $90^{\circ}$ was $0.76 \mathrm{~N} \cdot \mathrm{m}$. Changing the yaw angle from 45 to $0^{\circ}$ reduced the pitching moment by $37 \%$ at a pitch angle of $90^{\circ}$.

\subsubsection{Comparison of different rotor distances}

The thrust and pitching moment of a quad-rotor in a yaw direction of $0^{\circ}$ at different rotor distances are shown in Figs. 22 and 23.

For the thrust shown in Fig. 23, for the pitch angles of 60 


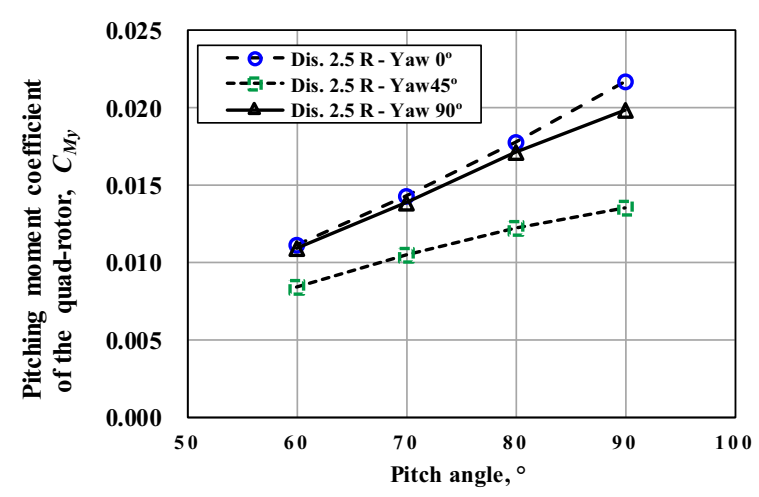

Fig. 22. Pitching moment of a quad-rotor at different yaw angles.

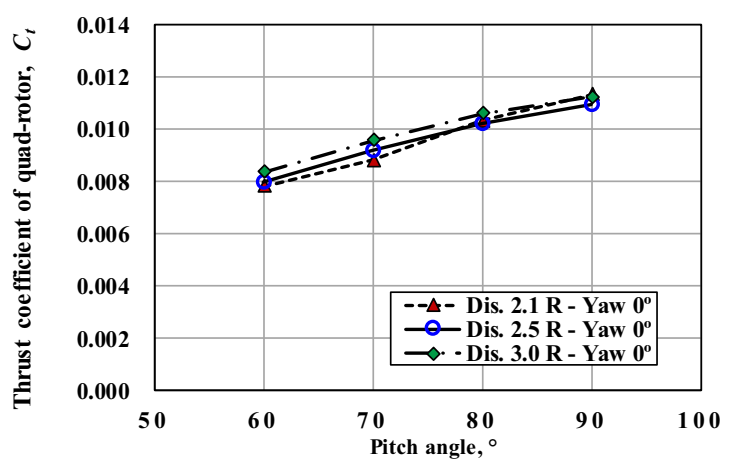

Fig. 23. Thrust of a quad-rotor at different rotors distance.

to $80^{\circ}$, the thrust for a rotor distance of $3.0 R$ was the largest among the rotors distances. However, at a pitch angle of $90^{\circ}$, the thrust was largest at a rotor distance of $2.1 R$. Based on a comparison between rotors distances of $2.5 R$ and $3.0 R$, the thrust at a distance of $3.0 R$ was larger than that at $2.5 R$ for all pitch angles.

For the pitching moment shown in Fig. 24, the moment was smallest at a rotor distance of $3.0 R$. At a pitch angle of $90^{\circ}$, the moment for the distance of $2.1 R$ was $0.76 \mathrm{~N} \cdot \mathrm{m}$, and the moment for a distance of $3.0 R$ was $0.66 \mathrm{~N} \cdot \mathrm{m}$. Increasing the rotor distance by $1.0 R$ reduced the moment generation by $13 \%$.

\subsection{Discussion}

\subsubsection{Effect of yaw direction of the body}

For the effect of the direction of motion on thrust, the thrust for a yaw direction of $45^{\circ}$, which was in a plus configuration with regard to the flow direction, was larger than that of the other two yaw angles. In the plus configuration in a free-stream, rotor wake interference occurred between only the two rotors at the centerline of the body. Therefore, a decrease in thrust occurred only at the rearmost rotor. Thus, the amount of the loss of total thrust of a quad-rotor was decreased. Moreover, the pitching moment was degraded in comparison to the $\mathrm{X}$ configuration. With the $\mathrm{X}$ configuration, the rotor wake interferes with the two rear rotors, and the thrust of the two rear rotors decreases. It is presumed that the effect of the rotor wake interference is more significant with the $\mathrm{X}$ configuration than with the plus configuration. The results were as expected.

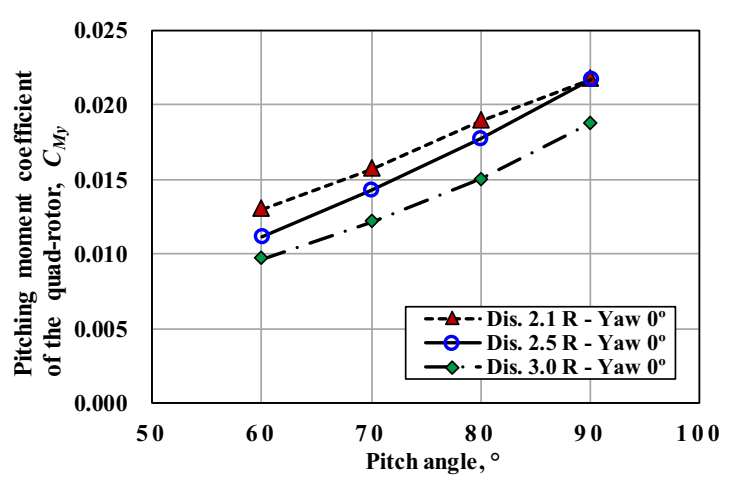

Fig. 24. Pitching moment of a rear rotor at different rotor distance.

For the $F x$, changing the direction of motion from a yaw angle of $0^{\circ}$ to an angle of $45^{\circ}$ reduced the $F x$ by $0.5 \mathrm{~N}$. Although the Fx differed between the yaw directions, $0.5 \mathrm{~N}$ is smaller than the sensor force range of $100 \mathrm{~N}$. Therefore, we were unable to determine the difference in $F x$ due to the yaw angle. We confirmed that $F x$ acts on the direction of the body, increasing the pitching moment in a uniform flow. The $F x$ varied from 3.4 to $4.1 \mathrm{~N}$ under the experimental conditions.

For the generation of pitching moment, the moment was smallest at a yaw angle of $45^{\circ}$. As mentioned with regard to the effect on the thrust, changing the configuration of the flow direction to a plus shape from an $\mathrm{X}$ shape seems to prevent rotor wake interference. Therefore, at a yaw angle of $45^{\circ}$, a loss of thrust is prevented as compared to an X configuration. Consequently, the difference in thrust among the rotors in a plus configuration is smaller than that in an X configuration. We assume that preventing a loss of thrust at the rear rotor decreases the pitching moment of a quad-rotor. At yaw angles of 0 and $90^{\circ}$ in an $\mathrm{X}$ configuration, the front rotor wake affects the two rear rotors. Moreover, the distance between the front and rear rotors in an X configuration is smaller than in a plus configuration. Therefore, the rotor wake interference decreases the rotor thrust of the two rear rotors and generates a more significant pitching moment than in a plus configuration.

Based on a comparison at yaw angles of 0 and $90^{\circ}$, differences in the forces and pitching moment were smaller than the force measured and the sensor range. Thus, the difference in rotor rotational direction in an $\mathrm{X}$ configuration does not seem to affect the forces on the body.

Through a comparison of different yaw angles, we concluded that a loss of thrust and generation of pitching moment were avoided by keeping the body configuration in a plus shape as compared to an X configuration. We presumed that the differences are caused by avoiding the rotor wake interface. The two rotor measurements clarified that increasing the rotor distance prevents a loss of rear rotor thrust. The quad-rotor measurement results are compatible with the presumed mechanism of pitching moment reduction with a plus configuration. For a flight with a uniform flow of the quadrotors, maintaining the body configuration in a plus shape is suitable for the free-stream direction. 


\subsubsection{Effects of rotor distances}

Increasing the rotor distance decreased the pitching moment at all pitch angles for a yaw angle of $0^{\circ}$, as shown in Fig. 24. For the two-rotor measurements, the rear rotor thrust decreased when reducing the rotor distance. In the quad-rotor model measurements, the rotor distance was changed from $2.1 R$ to $3.0 R$. Thus, the rear rotor was affected by the front rotor wake. Therefore, we assumed that the pitching moment caused by the difference in rotor thrust decreases when the rotor distance increases.

Although increasing the rotor distance degraded the pitching moment, the rotor distance range was closer than 3.0R. At a distance of over $5.0 R$, we infer that the rear rotor thrust reaches the thrust of the isolated rotor. To examine the rotor distance for a negligible loss of thrust, measurement using a longer rotor distance is required. However, the rotor distance was limited because of the size of the wind tunnel section. We concluded that expanding the rotor distance to under 5.0R decreases the pitching moment. Expanding the rotor distance is likely to decrease generation of head-up pitching moment of a quad-rotor during horizontal flight.

For the quad-rotor thrust, increasing the rotor distance from $2.1 R$ to $3.0 R$ increased thrust by $1 \mathrm{~N}$, except at a pitch angle of $90^{\circ}$. We presumed that the increase in thrust was caused eliminating the rotor wake interference, similar to the generation of the pitching moment. Although the trend regarding the thrust dependency on the rotor distance showed that increasing the rotor distance increases thrust, the difference in thrusts at $2.1 R$ and $3.0 R$ for pitch angles of 80 and $90^{\circ}$ did not agree with this trend. This inconsistency seems to be caused by the deterioration of measurement accuracy at high pitch angles. At a pitch angle of $90^{\circ}$, strong vibrations on the body were observed. Thus, the difference in thrust for different rotor distances at high pitch angles was not examined during the experiment.

\subsubsection{Effect of rotor flow interference on horizontal flight of quad-rotor UAVs}

The results of the two-rotor model measurements and quad-rotor model measurements showed that the effect of rotor wake interference is minimized as the rotor plane is tilted toward an upwind direction. Therefore, the effects of rotor wake interaction on the action force of a quad-rotor during horizontal flight become smaller as the flight speed increases. During a wind gust, while a UAV is hovering, the rotor flow interference can affect the reaction motion of the UAV body. Furthermore, a reduction in the pitching moment caused by an increase in rotor distance, and maintaining the direction of motion at $45^{\circ}$, were confirmed during the experiment. It should be noted, however, that increasing the rotor distance leads to an increase in body weight. Thus, a discussion regarding other factors is required to increase the rotor distance in order to reduce the pitching moment in the design process. Therefore, to decrease the pitching moment during horizontal flight, keeping the heading attitude toward the flow direction, as in a plus configuration, is suitable. This method for reducing the pitching moment is expected to increase the wind speed at which a quad-rotor UAVs can fly.

\section{Conclusions}

In this study, two experiments were conducted in a lowspeed wind tunnel. The study focuses on examining the effect of flow interaction on pitching moment on the quad-rotor with different rotor configurations in a uniform flow, which was intended to simulate the horizontal flight of a quad-rotor UAV. The first was a two-rotor measurement to examine the effects of rotor flow interaction on rotor thrust and pitching moment. The second was a quad-rotor measurement to examine the pitching moment on the body under different configurations.

For the two-rotor experiment, the rotor performance in terms of rotor wake interaction was assessed for three relative placements of the rotors: height, distance, and depth normal to the flow direction. In the rotor flow interaction between the two rotors, the front rotor performance was the same as that of an isolated rotor, which was not affected by the flow interaction. The rear rotor thrust depends on the relative position of the rotors due to the rotor flow interference.

For the quad-rotor measurement, it was confirmed that the pitching moment increased as the pitch angle increased. Moreover, pitching moment difference between the plus and X configurations as illustrated in Fig. 14 was confirmed. The plus configuration with regard to flow direction reduced the pitching moment by $37 \%$ compared to the $\mathrm{X}$ configuration. The effect of rotor flow interaction was reduced, and the pitching moment was decreased by making the body yaw direction the same as that of the free-stream. In addition, expanding the rotor distance from $2.1 R$ to $3.0 R$ reduced the pitching moment by $13 \%$ for the $\mathrm{X}$ configuration with regard to the flow direction. The experiment results verified the effective range of flow interaction at the relative position of each rotor. In terms of the pitch angle of the quad-rotor UAVs, the body tilts toward the upwind direction in horizontal flight. Thus, the effect of rotor flow interaction seems to be reduced in flight compared to the effect of that at a high pitch angle. However, there were differences in the pitching moment and thrust when changing the yaw direction and rotor distance at a pitch angle of $60^{\circ}$.

In consideration of the rotor placement of a quad-rotor during horizontal flight, we concluded that avoiding a flow interaction by maintaining the yaw direction in a plus configuration toward the flow, and expanding the rotor distance, reduces the pitching moment on the body as expected. Such a configuration has the potential to increase the wind speed at which a UAV can fly during outdoor operation.

\section{Acknowledgments}

This work was supported by the Grand-in Aid for the JSPS Research Fellow Grant, Number 16J02686. We thank the students in our laboratory for supporting the experiment: Masayoshi Kohno, Yasuhiro Kikuchi, Suguru Igarashi, Yota Konno, Kazuki Matsubara, and Himankar Sharma. 
Trans. Japan Soc. Aero. Space Sci., Vol. 61, No. 2, 2018

\section{References}

1) Mahony, R., Kumar, V., and Corke, P.: Multirotor Aerial Vehicles, IEEE Robotics and Automation Magazine, 32 (2012), pp. 20-32.

2) Stepniewski, W. Z.: A Simplified Approach to the Aerodynamic Rotor Interference of Tandem Helicopters, Proc. of West Coast American Helicopter Society Meeting, 1955, pp. 71-89.

3) Huston, R. J.: Wind-tunnel Measurements of Performance, Blade Motions, and Blade Air Loads for Tandem-Rotor Configurations with and without Overlap, NASA TN-D-1971, 1963.

4) Johnson, W.: Helicopter Theory, Dover Publications, New York, 1994, pp. 142-146.

5) Harris, F. D.: Technical Note: Twin Rotor Hover Performance, J. Am. Helicopter Soc., 44 (1999), pp. 34-37.

6) Bagai, A. and Leishman, J. G.: Free-Wake Analysis of Tandem, TiltRotor and Coaxial Rotor Configurations, J. Am. Helicopter Soc., 41 (1996), pp. 196-207.

7) Yoon, S., Lee, H. C., and Pulliam, T. H.: Computational Analysis of Multi-rotor Flows, AIAA Paper 2016-0812, 2016.

8) Yeo, H. and Johnson, W.: Performance and Design Investigation of Heavy Lift Tilt-Rotor with Aerodynamic Interference Effects,
J. Aircraft, 46 (2009), pp. 1231-1239.

9) Ramasamy, M.: Hover Performance Measurements toward Understanding Aerodynamic Interference in Coaxial, Tandem and Tilt Rotors, J. Am. Helicopter Soc., 60 (2015), pp. 032005-1-17.

10) Theys, B., Hendrick, P., and Schutter, D. J.: Experimental and Numerical Study of Micro-Aerial-Vehicle Propeller Performance in Oblique Flow, J. Aircraft, 54 (2017), pp. 1076-1084.

11) Russell, C. R., Jung, J., Willink, G., and Glasner, B.: Wind Tunnel and Hover Performance Test Results for Multicopter UAS Vehicles, Proc. American Helicopter Society, 72nd American Helicopter Soc. International Annual Forum and Technology Display, West Palm Beach, FL, USA, 2016.

12) Hwang, J. Y., Jung, M. K., and Kwon, O. J.: Numerical Study of Aerodynamic Performance of a Multirotor Unmanned-Aerial-Vehicle Configuration, J. Aircraft, 52 (2015), pp. 839-846.

13) Leishman, J. G.: Principles of Helicopter Aerodynamics, 2nd Ed., Cambridge University Press, New York, 2006, pp. 95-100.

S. Saito

Associate Editor 